\title{
Therapist-Guided Internet-Based Treatments for Loneliness: A Randomized Controlled Three-Arm Trial Comparing Cognitive Behavioral Therapy and Interpersonal Psychotherapy
}

\author{
Anton Källa Malin Bäck ${ }^{a} \quad$ Camilla Welin $^{a} \quad$ Hanna Åman $^{a} \quad$ Rasmus Bjerkander $^{a}$ \\ Matilda Wänman ${ }^{a}$ Tomas Lindegaard ${ }^{a}$ Matilda Berg ${ }^{a}$ Hajdi Moche $^{a}$ \\ Roz Shafran ${ }^{b}$ Gerhard Andersson ${ }^{a, c, d}$ \\ a Department of Behavioral Sciences and Learning, Linköping University, Linköping, Sweden; ${ }^{b}$ UCL Great Ormond \\ Street Institute of Child Health, London, UK; ' ${ }^{C}$ Department of Biomedical and Clinical Sciences, Linköping University, \\ Linköping, Sweden; ${ }^{\text {d}}$ Department of Clinical Neuroscience, Karolinska Institute, Stockholm, Sweden
}

\author{
Keywords \\ Loneliness · Internet treatment · Cognitive behavioral \\ therapy · Interpersonal psychotherapy · Guided self-help
}

\begin{abstract}
Introduction: Chronic loneliness has been linked to many adverse outcomes, including mental health problems. Psychological treatment of loneliness can be effective, but the evidence base is limited. Objective: To investigate the efficacy of 2 internet-based interventions based on cognitive behavioral therapy (ICBT) and interpersonal psychotherapy (IIPT) relative to a wait-list control group and each other. Methods: A total of 170 participants were recruited and randomized to either 9 weeks of ICBT $(n=68)$, IIPT $(n=68)$, or a wait-list condition $(n=34)$. The primary outcome was loneliness, measured using the UCLA Loneliness Scale before, during, and after treatment. Secondary measures of psychiatric disorders and quality of life were administered before and after treatment. Follow-up was conducted 4 months after the treatment had ended. Primary outcome data were analyzed using growth curve modeling. Secondary outcomes
\end{abstract}

karger@karger.com www.karger.com/pps

Karger $\frac{1}{\%}$
(C) 2021 The Author(s)

Published by S. Karger AG, Basel

This is an Open Access article licensed under the Creative Commons Attribution-NonCommercial-4.0 International License (CC BY-NC) (http://www.karger.com/Services/OpenAccessLicense), applicable to the online version of the article only. Usage and distribution for commercial purposes requires written permission. were analyzed using robust regression models. The trial was preregistered (ClinicalTrials.gov ID: NCT03807154). Results: The ICBT condition had a significantly greater impact on loneliness compared to the wait-list and IIPT conditions. Effect sizes were moderate to large (Cohen $d=0.71$ ) compared to the wait-list and moderate $(d=0.53)$ compared to IIPT. The IIPT condition did not differ significantly from the wait-list. Both active treatments led to significant increases in quality of life. Only the ICBT group had significantly lower symptoms of depression and generalized anxiety compared to the waitlist group. Treatment gains were maintained but not improved at follow-up. Conclusions: ICBT can be an efficacious option for alleviating loneliness. The IIPT intervention was not as effective.

(c) 2021 The Author(s)

Published by S. Karger AG, Basel

\section{Introduction}

Loneliness is defined as an aversive emotional reaction resulting from a perceived deficiency in a person's social network [1]. It can have detrimental effects on physical 
health, e.g., coronary heart disease and stroke [2]. The percentage of the population that often or always experiences loneliness was $6 \%$ in a study of adults in the UK [3] and $11-14 \%$ in a study of older adults over the age of 65 years in Sweden [4]. Frequent experiences of loneliness have been linked to increased psychiatric symptoms such as depression [5], social anxiety [6,7], and suicide attempts [8]. There is limited research on how to best help people who experience loneliness. Psychological interventions could be a viable option, with a meta-analysis suggesting that cognitive behavioral therapy (CBT) may be effective [9]. The rationale behind using CBT as a means of reducing loneliness stems in part from the connection between this state and maladaptive cognitive processes [10]. Additionally, behavioral patterns such as an overreliance on avoidance or a lack of social skills are important to consider [11]. Examples of prior CBT interventions for loneliness are described in the online supplementary material (for all online suppl. material, see www. karger.com/doi/10.1159/000516989).

The current trial investigated the effects of 2 internetbased interventions based on CBT (ICBT) or interpersonal psychotherapy (IPT). The ICBT condition served as a replication and extension of a previous pilot trial [12]. The internet-based IPT condition (referred to as IIPT) aimed to address interpersonal psychological processes not covered directly in ICBT. IPT focuses on the link between mood and interpersonal events, proposing that psychopathology arises and is maintained as a result of adverse changes in one's social network and subsequent functional deficits in these relationships [13]. Meta-analyses support the efficacy of IPT for common mental health problems [14]. Additional reasons for targeting interpersonal mechanisms related to loneliness are described in the online supplementary materials. With the 2 different approaches, we explored the specific impact of addressing intrapersonal factors (i.e., maladaptive behavioral and cognitive patterns) and interpersonal factors (i.e., adverse interpersonal events and the subsequent lack of social support). As the treatment programs were either novel or only evaluated once, we also compared them against a wait-list control group.

Our hypotheses were that: (1) both active treatments would show a significant reduction in loneliness compared to a wait-list control group and these reductions would be maintained 4 months after the conclusion of the interventions and (2) there would be a significant increase in quality of life and a significant reduction in symptoms of psychiatric disorders for both treatments compared to the control group. We were not able to form a hypothesis about the differential impact of the 2 treatments. However, we conducted exploratory analyses to explore differences in loneliness, psychiatric symptoms, and quality of life between the 2 active interventions.

\section{Materials and Methods}

\section{Design}

The trial was registered at ClinicalTrials.gov (ID: NCT03807154). The recruitment took place on 2 occasions, i.e., one in January 2019 $(n=116)$ and one in January $2020(n=54)$. Randomization was conducted using a true random number generator website (random. org). Participants were randomized and assigned to each condition by 2 external parties not involved in other aspects of this study, using an anonymized 8-character ID (e.g., 1234abcd). The ratio used for the randomization was 2:2:1 (treatment:treatment:control). This trial is reported according to the CONSORT guidelines [15].

Participants, Recruitment, and Inclusion/Exclusion Criteria

Recruitment was conducted during 2-week intakes in January 2019 and 2020 (see online suppl. materials for further details). The web page (referred to as platform) on which the intervention was hosted is encrypted and has hosted similar studies [16]. Participants provided informed consent and were asked to complete a screening survey. They were also called for a structured interview using the MINI neuropsychiatric interview 7.0 [17]. Demographic characteristics and psychiatric diagnoses as indicated by the MINI interview are presented in online supplementary Table 1. A flowchart of the recruitment procedure is displayed in Figure 1.

The inclusion criteria were: (1) suffering and distress as a result of the subjective experience of loneliness; (2) age 18 years or older; (3) ability to write, speak, and read Swedish; (4) access to the internet and a computer/smartphone; (5) if applicable, a stable dose of psychotropic medication and no planned changes; and (6) willingness to participate in this study regardless of the condition to which they were randomized. The judgement of criterion (1) was made by the participants themselves, as there are no known clinical cutoffs for the primary outcome instrument. Participants were excluded if they: (1) were currently undergoing another psychological treatment; (2) had a previously diagnosed personality syndrome (as diagnosed by a licensed healthcare professional); (3) reported ongoing drug abuse; and (4) psychiatric comorbidities that could not be managed within the frame of the study, e.g., acute suicidal intent or anorexia nervosa. Other psychiatric diagnoses, either previously diagnosed or as indicated by the MINI interview, did not serve as a reason for exclusion as long as they were not judged to interfere with the intervention or require another treatment (such as severe OCD). However, in the case of comorbid conditions, participants were asked to identify their primary concern. Participants endorsing comorbid conditions such as depression or anxiety disorders, rather than loneliness, as their primary concern were not included in this study. The decision on inclusion/exclusion was based on the self-report of participants, results from the questionnaires, the structured interview, and a clinical evaluation by the interviewer. Cases were discussed in meetings staffed by all of the interviewers and 2 registered clinical psychologists, one of whom was the principal investigator (G.A.). 


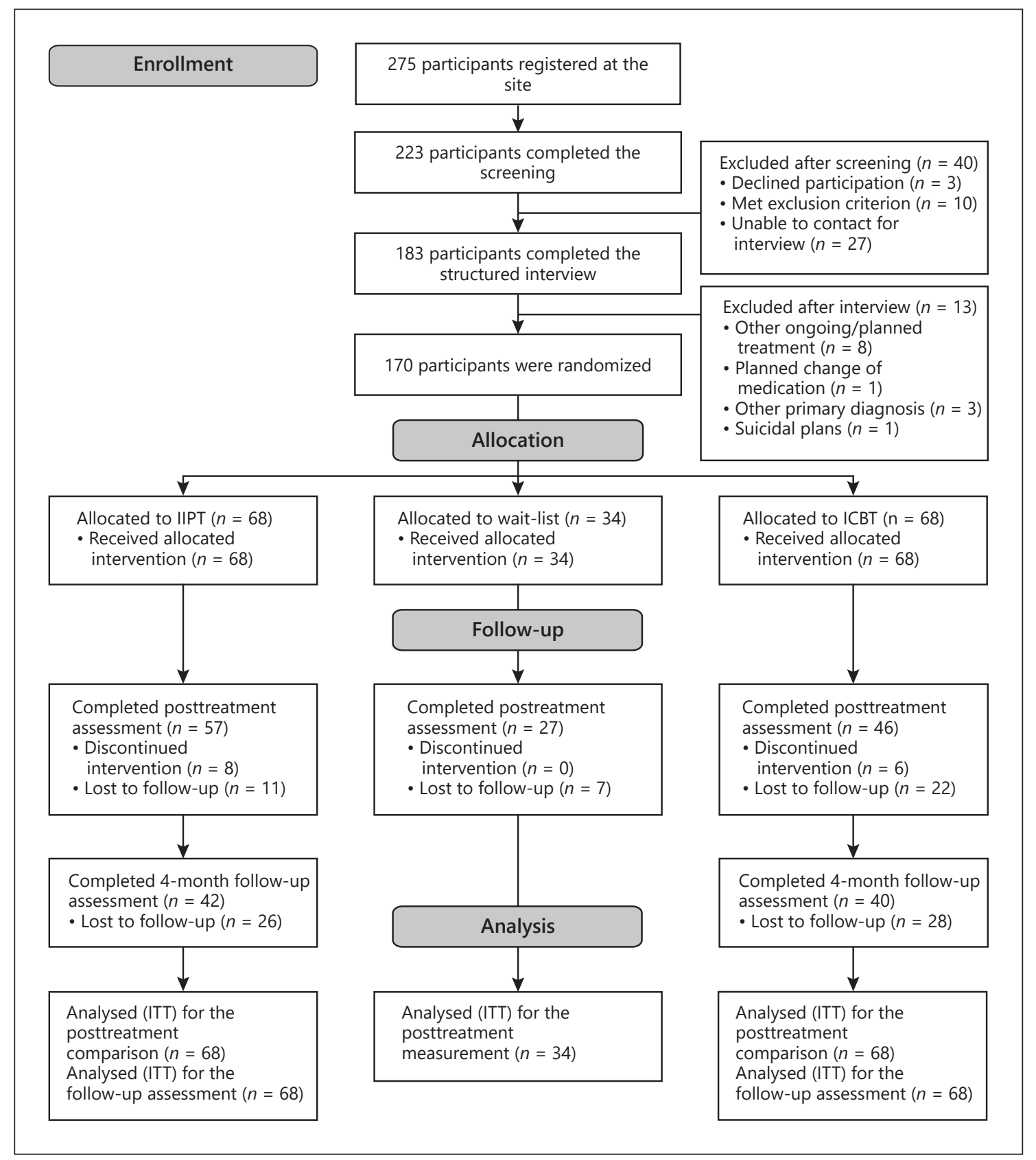

Fig. 1. Flowchart of the recruitment and assessments during this study.

The decision on inclusion/exclusion was made by the team, with the principal investigator having the final responsibility for the decision.

\section{Procedure}

Eight students from the clinical psychologist program at Linköping University and 1 licensed clinical psychologist served as assessors for this study. All of them were trained in using MINI 7.0 and the additional questions asked during the structured interview. In total, 8 therapists were involved as therapists during the 2 intakes. More information about the therapists and their role can be found in the online supplementary material.

\section{Conditions}

The 2 interventions each consisted of 9 modules that contained text, pictures, and interactive assignments related to the theme of loneliness and how to deal with it. Details of the content of the interventions are available in the online supplementary material. An outline of all of the modules can be viewed in online supplementary Tables 2 and 3. 
All of the participants in the treatment groups received weekly feedback on their work on a set weekday (Monday) by their assigned therapist. They could contact the therapist when needed using the platform's messaging system and receive a response within $24 \mathrm{~h}$ on work days. The therapists had no restrictions on the amount of time that they would allocate to help the participants (for further details about the therapists, see the online suppl. material).

Subjects in the wait-list control group received information about the measurements and were told that they would receive treatment after 11 weeks. Participants in the control group had the option to contact the research group via the study email address if needed (e.g., if they wanted additional information about this study). Interactions with the control group were very infrequent overall and were handled by a person who had no contact with the treatment groups.

\section{Measures}

The primary outcome of loneliness was measured using the UCLA Loneliness Scale, Version 3 (ULS-3) [18]. This questionnaire consists of 20 items for which the participants are asked to indicate the frequency of experiences linked to loneliness. Responses are made on a 4-point Likert scale (scored between 1 and 4). The scores from all items are summed up with a possible range between 20 and 80 , with higher scores indicating more frequent loneliness. The instrument has been shown to have an internal consistency ranging from $\alpha=0.89$ to $\alpha=0.94$ and a 1 -year test-retest reliability of 0.73 during validation of the questionnaire. In the current study the internal consistency was $\alpha=0.87$. The primary outcome measure was administered before treatment, every 2 weeks during the intervention, and after treatment, as well as 4 months after the conclusion of the intervention. Secondary outcomes included symptoms of depression measured with the Patient Health Questionnaire-9 (PHQ9) [19], symptoms of social anxiety measured with the Social Interaction Anxiety Scale (SIAS) [20], symptoms of generalized anxiety measured using the Generalized Anxiety Disorder 7-item scale (GAD-7) [21], and quality of life measured with the Brunnsviken Brief Quality of Life Scale (BBQ) [22]. All secondary measures were included before and after treatment, as well as 4 months after the conclusion of the intervention. Three other measures were administered to measure factors related to the efficacy of the intervention: credibility and expectancy measured with the 6-item Credibility and Expectancy Questionnaire (CEQ) [23], satisfaction with the treatment measured with the Client Satisfaction Questionnaire 8 -item scale (CSQ) [24], and working alliance measured with the 12-item version of the Working Alliance Inventory (WAI) [25]. The CEQ and the WAI were administered during week 3 of the intervention and the CSQ only after treatment. Additionally, 4 questions were asked to gain insight into which factors the participants considered to be of importance for reducing their loneliness. The items can be viewed in the online supplementary material.

\section{Statistical Analysis}

Statistical analyses were conducted using SPSS 25 and Mplus 8.4 [26]. The a level was set at 0.05 , though all three-way comparisons made use of a Bonferroni-corrected a level of 0.0166 to ac- count for multiple comparisons. For analyses of the three-way comparisons, $p$ values were corrected to allow for the same interpretation as analyses with the standard a level. Corrections were made by multiplying the $p$ values by the number of comparisons (i.e., 3). Confidence intervals are reported at 95\% for two-way comparisons and at $99 \%$ for three-way comparisons (the closest available level reported in the statistical software). Testing was two-tailed. Data were handled according to the intention-to-treatprinciple. Missing data was handled using maximum likelihood estimation, i.e., 1 of 2 recommended approaches for dealing with missing data under the assumption that missingness is not related to the would-be value (i.e., missing at random) [27].

For the first aim regarding differences in the primary outcome measure, we used a latent growth curve framework [28]. A conditional linear growth model was used to measure the interaction between slope and condition along with estimates of heterogeneity for the intercept and slope. Differences between the 2 active conditions and the wait-list were investigated using 2 dummy-coded predictors $(0=$ wait-list and $1=\mathrm{IIPT} / \mathrm{ICBT})$. A separate model investigated the comparison between the 2 active conditions (coded as $-0.5=$ IIPT, $0=\mathrm{WL}$, and $0.5=$ ICBT, with an additional contrast comparing the combined treatment group vs. the wait-list). Further details about the primary outcome model are available in the online supplementary material. The statistical analyses for the secondary outcomes are described in the online supplementary material.

To investigate change during the follow-up period in the primary outcome measure, we extended the model outlined above to include an additional time piece, known as a piecewise model, allowing for estimation of specific phases of the treatment [29] (i.e., treatment phase and follow-up phase). This model only made use of data from participants in the active treatment groups $(n=136)$. The variance of the slopes was fixed at 0 . The analysis was conducted using the grouping option in Mplus, meaning that separate slopes were calculated for the 2 groups, allowing for inferences about how the respective groups changed during the follow-up phase.

As recommended by Feingold [30], standardized effect sizes were estimated using the estimated mean difference at the time point of interest (after treatment) divided by the pooled standard deviation at pre-treatment (i.e., Cohen's $d$ ). Within-group effect sizes were calculated with the pooled estimated standard deviations from both time points.

\section{Results}

\section{Demographic Characteristics}

Overall, the sample consisted of $75.9 \%$ women and had a mean age of 47.5 years $(\mathrm{SD}=16.4)$. Furthermore, $69.4 \%$ had a university degree and $76 \%$ reported their civil status as either single $(52.4 \%)$, divorced $(17.1 \%)$, or widowed $(6.5 \%)$. There were no significant differences between the groups at the pretreatment assessment for any of the outcomes measures or the demographic characteristics (all $p$ $>0.078)$. Further details regarding the demographic characteristics of the sample with between-group comparisons are presented in online supplementary Table 1. 


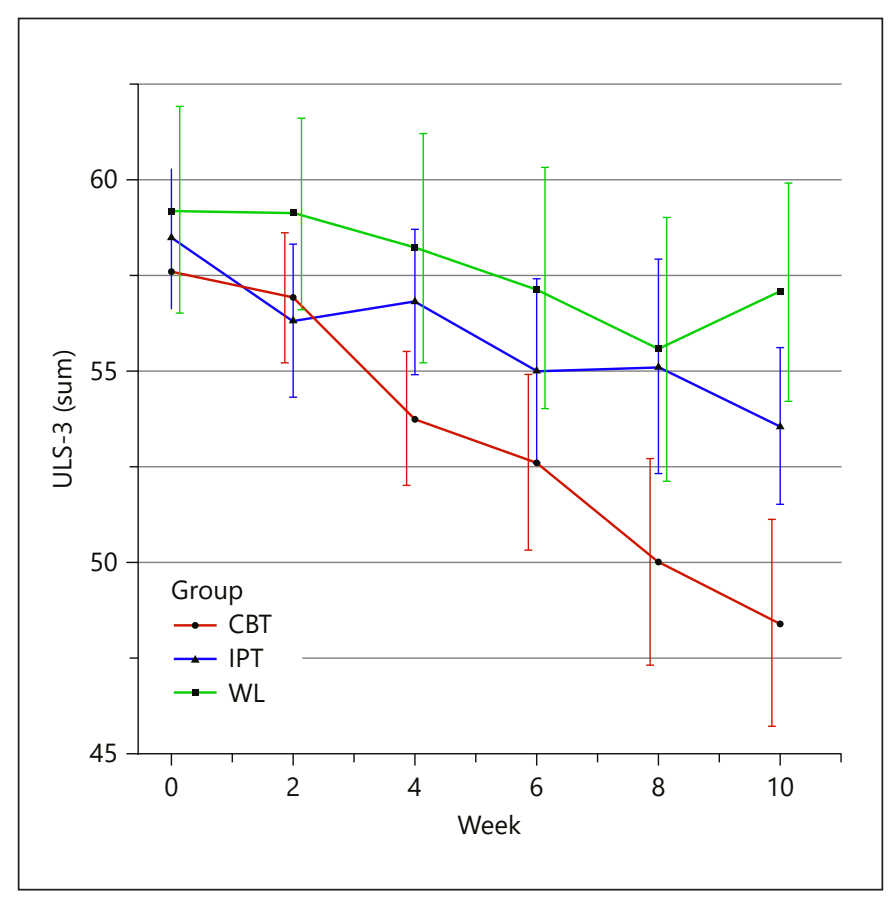

Fig. 2. Observed means for all conditions during the treatment, with $95 \%$ CI.

\section{Effect of the Interventions}

Activity statistics can be seen in online supplementary in Table 4. The observed means and SD for the outcome measures can be viewed in online supplementary Table 5 . The observed change in loneliness during the treatment phase can be seen in Figure 2. Sensitivity analyses and calculations of clinically significant change are available in the online supplementary material.

\section{Treatment Conditions Relative to the Control Group after Treatment}

Estimates for the fixed and random effects along with model fit indices of the growth curve model for the primary outcome measure are presented in online supplementary Table 6 . The model investigating change in loneliness levels indicated significant heterogeneity in the initial level $(b=43.97 ; 95 \%$ CI 32.98-54.95; SE $=5.60 ; p<$ $0.0001)$ and slope $(b=0.34 ; 95 \%$ CI $0.16-0.51 ; \mathrm{SE}=0.09$; $p<0.0001)$. For the ratings of loneliness, the analysis revealed a significant difference favoring the ICBT condition as compared to the control group during the treatment $(b=-5.22 ; 99 \% \mathrm{CI}-9.61$ to -0.83 ; $\mathrm{SE}=1.70 ; p=$ $0.006 ; d=0.71)$. The IIPT condition did not show a statistically significant decrease in loneliness during the treatment period relative to the wait-list condition $(b=$

Guided Internet-Based Treatments for Loneliness
-1.36 ; $99 \%$ CI -4.19 to 1.48 ; $\mathrm{SE}=1.44 ; p=1 ; d=0.18$ ). The results for the secondary outcomes can be seen in the online supplementary material.

\section{Comparisons between the Active Conditions after Treatment}

The analysis of the loneliness measure showed that participants in the ICBT group had a significantly larger reduction compared to the IIPT group $(b=-3.87 ; 99 \%$ CI -7.28 to $-0.45 ; \mathrm{SE}=1.32 ; p=0.012 ; d=0.53)$.

\section{Follow-Up Four Months after the Posttreatment Assessment}

The analysis of the posttreatment to follow-up time piece indicated that the gains were maintained at followup. The results indicated a nonsignificant increase in ratings of loneliness for the participants in the ICBT group $(b=1.66 ; 95 \% \mathrm{CI}-0.57$ to $3.88 ; \mathrm{SE}=1.14 ; p=0.144)$. The estimated within-group effect size from pretreatment to follow-up was $d=0.81$. During the same period, the model indicated a nonsignificant decrease for the IIPT group $(b=-1.25 ; 95 \% \mathrm{CI}-2.97$ to $0.47 ; \mathrm{SE}=0.88 ; p=0.155)$. The effect size from pretreatment to follow-up was $d=0.70$. Analyses of the secondary measures at follow-up can be found in the online supplementary material.

\section{Discussion}

We investigated the efficacy of 2 internet-based interventions targeting loneliness with guidance from a therapist. The results showed that the ICBT program led to a significant reduction of loneliness compared to the waitlist control group, whereas the IIPT program did not. Furthermore, the ICBT condition also led to a significantly larger decrease in the primary outcome measure during the treatment period compared to the IIPT condition. The results from the 4-month follow-up indicated no significant changes in loneliness between the posttreatment and follow-up assessments for either the ICBT group or the IIPT group. Both active conditions exhibited large gains in self-rated quality of life. The ICBT group also had significantly lower ratings of depressive symptoms and generalized anxiety than the control group at the posttreatment assessment. There were no significant differences between the active conditions at follow-up for any of the secondary outcome measures. The findings extend the sparse number of interventions for loneliness by evaluating 2 novel psychological treatments in a methodologically sound design. 
The significantly steeper decline of loneliness in the ICBT condition compared to the control group was in line with our hypothesis. The effect size for this comparison was moderate $(d=0.71)$, which is similar to the one found in our previous pilot trial [21]. The effect size in the current study was larger than an intervention on older adults utilizing CBT elements [31]. The withingroup effect size in the CBT condition is similar to that found in an intervention based on a social identity approach (Groups 4 Health) [32] (within-group $d=0.99$ for the CBT condition and $d=1.04$ for the Groups 4 Health condition), although the latter does not present a between-group effect size to allow comparisons taking the control group into account. Even if the instrument used for measuring loneliness has no clinical cut-off, the mean score after treatment can be interpreted as indicating a mean frequency of loneliness experiences (e.g., lacking companionship) ranging between "rarely" and "sometimes." The mean score after treatment was roughly 4 scale points above the reported mean in a representative normal American sample [33], suggesting that the participants on average still had a somewhat elevated level of loneliness compared to the general population.

The current study is important for multiple reasons. First, it adds to the increasing number of studies indicating that methods and techniques found under the CBT umbrella can be effective when targeting loneliness [34, 35]. While other psychological interventions can reduce loneliness [32], the results from this trial and others employing similar elements and theoretical assumptions $[36,37]$ suggest that CBT indeed can be considered a prime candidate for alleviating loneliness as previously suggested [9]. This conclusion is strengthened by the finding that ICBT also performed favorably compared to another active, credible psychological treatment. However, given that we tested the impact of 2 treatment packages with different treatment techniques, we cannot state which components were most important or whether nonspecific factors such as receiving attention were equally or more important [38]. Secondly, comparisons between active treatments are important in informing treatment selection in a field where there are no evidence-based guidelines. In addition, as the effects of ICBT were replicated in this trial, we believe that ICBT targeting loneliness could be a way of providing help to this population on a broader scale, as internet-based psychological treatments have been proposed as a way to deliver evidence-based treatments to underserved populations [39]. The findings suggest that treatment gains were maintained but there were no further reductions in loneliness at the follow-up assessment. This is not uncommon among internet-delivered treatments [40] and is something that should be investigated further. Another factor is the specificity of the intervention given that we also saw reductions in symptoms of psychiatric disorders commonly linked to loneliness (e.g., depression). The causal link between reductions of symptoms of these disorders and the experience of loneliness remains unclear but could be important in understanding the mechanisms involved in reducing loneliness.

The lack of an effect of IIPT on loneliness was unexpected and there are several possible explanations for this. Although the program was created with the help of an experienced IPT therapist and supervisor who also participated in the planning and supervision of the trial, the lack of prior models and empirical studies on the topic of loneliness in the context of IPT makes it hard to draw conclusions about the validity of the conceptualization we developed. Furthermore, IPT as an internet-based therapy has only been tested twice to our knowledge [41, 42 ], and it is not clear whether IPT for depression and anxiety disorders [14] can be delivered as internet treatments. It should be noted that IIPT led to a large effect relative to the control group for the quality-of-life measure and a moderate-to-large within-group effect size for loneliness at follow-up.

The strengths of this study include its randomized, controlled design with 2 active, credible treatment groups. This allows for comparisons both between those participants receiving treatment and those who did not in the initial stage, but also between 2 bona fide psychological treatments with researchers from different theoretical orientations. Additionally, measures of treatment credibility, working alliance, and satisfaction with the treatment helped to account for the potential of confounding variables when comparing the efficacy of the 2 active conditions.

With these in mind, there are additional limitations to consider when interpreting the results. First, the education level in the sample was higher than in the general population, with almost $70 \%$ having a university degree. Second, Sweden has a high degree of computer literacy, which could imply that the effects would be weaker in countries in which the population uses computers and smartphones less frequently. Third, participants were recruited from the general public, which most likely resulted in more motivated participants than in clinic-based settings. In addition, $76 \%$ of the sample were women while gender differences in loneliness have been small in 
previous research [43]. With regards to the sample, a sensitivity analysis revealed lesser treatment gains for participants with a probable psychiatric diagnosis. This suggests that the intervention could be less useful in psychiatric settings. Another limitation is the lack of a standardized loneliness criterion for inclusion. It is difficult to estimate how representative the current sample was compared to the population of lonely individuals at large. It should be noted that all of the participants considered loneliness to be their primary concern, although establishing normative scores and standardized inclusion criteria would help when comparing studies recruiting participants on the basis of feeling lonely. Similarly, the lack of a criterion for treatment completion is something to address going forward as such a criterion aids the ability to compare studies and synthesize findings in metaanalyses. Finally, we did not include a control group at the follow-up, which limits the conclusions about long-term effects and rules out other explanations such as regression to the mean [44].

In conclusion, the results are in line with previous findings and suggest that a CBT-based intervention is an efficacious way of reducing feelings of loneliness. Participants in the IIPT condition did not benefit significantly in terms of reductions in loneliness, but a moderate increase in quality of life was observed. The results help to further our understanding of the efficacy of psychological interventions targeting loneliness and, more specifically, suggest that ICBT is a valid option to consider for this population. With remote health care alternatives increasingly viewed as a viable option among clinicians and patients alike [45], expanding the current treatments with an intervention for loneliness is a relevant and accessible option for an underserved group.

\section{Acknowledgement}

The authors wish to thank George Vlaescu for his role as webmaster in the trial. All of the research at the Great Ormond Street Hospital NHS Foundation Trust and the UCL Great Ormond Street Institute of Child Health was made possible by the NIHR Great Ormond Street Hospital Biomedical Research Centre. The views expressed are those of the authors and not necessarily those of the NHS, the NIHR, or the Department of Health.

\section{Statement of Ethics}

All of the participants provided informed consent while applying for participation in this study. This study was approved by the regional ethics committee and conducted in accordance with the World Medical Association Declaration of Helsinki.

\section{Conflict of Interest Statement}

The authors have no conflict of interests to declare.

\section{Funding Sources}

This study was funded in part by a professor's grant to G. Andersson by Linköping University. Linköping University had no role in the preparation of data or this paper.

\section{Author Contributions}

A. Käll, R. Shafran, and G. Andersson conceived this trial. A. Käll, M. Bäck, R. Shafran, and G. Andersson designed this trial. A. Käll, M. Bäck, C. Welin, H. Åman, R. Bjerkander, M. Wänman, T. Lindegaard, M. Berg, and H. Moche conducted this study. A. Käll conducted the statistical analyses and drafted this paper. All of the authors read and approved the first and the final iterations of this paper.

\section{References}

1 Peplau LA, Perlman D. Loneliness: a sourcebook of current theory, research and therapy. New York, NY, US: Wiley; 1982.

2 Valtorta NK, Kanaan M, Gilbody S, Ronzi S, Hanratty B. Loneliness and social isolation as risk factors for coronary heart disease and stroke: systematic review and meta-analysis of longitudinal observational studies. Heart. 2016 Jul;102(13):1009-16.

3 Victor CR, Yang K. The prevalence of loneliness among adults: a case study of the United Kingdom. J Psychol. 2012 Jan-Apr;146(1-2): 85-104.

4 Dahlberg L, Agahi N, Lennartsson C. Lonelier than ever? Loneliness of older people over two decades. Arch Gerontol Geriatr. 2018;75:96103.
5 Cacioppo JT, Hawkley LC, Thisted RA. Perceived social isolation makes me sad: 5 -year cross-lagged analyses of loneliness and depressive symptomatology in the Chicago Health, Aging, and Social Relations Study. Psychol Aging. 2010 Jun;25(2):453-63.

6 Lim MH, Rodebaugh TL, Zyphur MJ, Gleeson JF. Loneliness over time: the crucial role of social anxiety. J Abnorm Psychol. 2016 Jul; 125(5):620-30

7 Maes M, Nelemans SA, Danneel S, Fernández-Castilla B, Van den Noortgate W, Goossens $\mathrm{L}$, et al. Loneliness and social anxiety across childhood and adolescence: multilevel meta-analyses of cross-sectional and longitudinal associations. Dev Psychol. 2019 Jul; 55(7):1548-65.
8 Solmi M, Veronese N, Galvano D, Favaro A, Ostinelli EG, Noventa V, et al. Factors associated with loneliness: An umbrella review of observational studies. J Affect Disord. 2020 Jun;271:131-8.

9 Masi CM, Chen HY, Hawkley LC, Cacioppo JT. A meta-analysis of interventions to reduce loneliness. Pers Soc Psychol Rev. 2011 Aug; 15(3):219-66.

10 Spithoven AW, Bijttebier P, Goossens L. It is all in their mind: A review on information processing bias in lonely individuals. Clin Psychol Rev. 2017 Dec;58:97-114.
Guided Internet-Based Treatments for Loneliness
Psychother Psychosom 2021;90:351-358 DOI: $10.1159 / 000516989$ 
11 Käll A, Shafran R, Lindegaard T, Bennett S, Cooper Z, Coughtrey A, et al. A common elements approach to the development of a modular cognitive behavioral theory for chronic loneliness. J Consult Clin Psychol. 2020 Mar; 88(3):269-82.

12 Käll A, Jägholm S, Hesser H, Andersson F, Mathaldi A, Norkvist BT, et al. Internet-based cognitive behavior therapy for loneliness: A pilot randomized controlled trial. Behav Ther. 2020 Jan;51(1):54-68.

13 Lipsitz JD, Markowitz JC. Mechanisms of change in interpersonal therapy (IPT). Clin Psychol Rev. 2013 Dec;33(8):1134-47.

14 Cuijpers P, Donker T, Weissman MM, Ravitz $\mathrm{P}$, Cristea IA. Interpersonal Psychotherapy for Mental Health Problems: A Comprehensive Meta-Analysis. Am J Psychiatry. 2016 Jul;173(7):680-7.

15 Boutron I, Moher D, Altman DG, Schulz KF, Ravaud P; CONSORT Group. Extending the CONSORT statement to randomized trials of nonpharmacologic treatment: explanation and elaboration. Ann Intern Med. 2008 Feb;148(4):295-309.

16 Vlaescu G, Alasjö A, Miloff A, Carlbring P, Andersson G. Features and functionality of the Iterapi platform for internet-based psychological treatment. Internet Interv. 2016 Oct; $6: 107-14$

17 Lecrubier Y, Sheehan DV, Weiller E, Amorim P, Bonora I, Harnett Sheehan K, et al. The Mini International Neuropsychiatric Interview (MINI): A short diagnostic structured interview - Reliability and validity according to the CIDI. Eur Psychiatry. 1997;12(5):22431.

18 Russell DW. UCLA Loneliness Scale (Version 3): reliability, validity, and factor structure. J Pers Assess. 1996 Feb;66(1):20-40.

19 Kroenke K, Spitzer RL, Williams JB. The PHQ-9: validity of a brief depression severity measure. J Gen Intern Med. 2001 Sep;16(9): 606-13.

20 Mattick RP, Clarke JC. Development and validation of measures of social phobia scrutiny fear and social interaction anxiety. Behav Res Ther. 1998 Apr;36(4):455-70.

21 Spitzer RL, Kroenke K, Williams JB, Löwe B. A brief measure for assessing generalized anxiety disorder: the GAD-7. Arch Intern Med. 2006 May;166(10):1092-7.

22 Lindner P, Frykheden O, Forsström D, Andersson E, Ljótsson B, Hedman E, et al. The Brunnsviken Brief Quality of Life Scale (BBQ): Development and Psychometric Evaluation. Cogn Behav Ther.2016 Apr;45(3):18295.
23 Devilly GJ, Borkovec TD. Psychometric properties of the credibility/expectancy questionnaire. J Behav Ther Exp Psychiatry. 2000 Jun;31(2):73-86.

24 Attkisson CC, Zwick R. The client satisfaction questionnaire. Psychometric properties and correlations with service utilization and psychotherapy outcome. Eval Program Plann. 1982;5(3):233-7.

25 Andrusyna TP, Tang TZ, DeRubeis RJ, Luborsky L. The factor structure of the working alliance inventory in cognitive-behavioral therapy. J Psychother Pract Res. 2001;10(3): 173-8.

26 Muthén BO, Muthén LK. Mplus user's guide. Los Angeles: Muthén \& Muthén; 2015.

27 Enders CK. Applied missing data analysis. New York, NY, US: Guilford Press; 2010.

28 Hesser $\mathrm{H}$. Modeling individual differences in randomized experiments using growth models: recommendations for design, statistical analysis and reporting of results of internet interventions. Internet Interv. 2015 May;2(2): $110-20$.

29 Raudenbush SW, Bryk AS. Hierarchical linear models: applications and data analysis methods. Thousand Oaks, CA, US: Sage Publications; 2002 .

30 Feingold A. Effect sizes for growth-modeling analysis for controlled clinical trials in the same metric as for classical analysis. Psychol Methods. 2009 Mar;14(1):43-53.

31 Cohen-Mansfield J, Hazan H, Lerman Y, Shalom V, Birkenfeld S, Cohen R. Efficacy of the I-SOCIAL intervention for loneliness in old age: lessons from a randomized controlled trial. J Psychiatr Res. 2018 Apr;99(1):69-75.

32 Haslam C, Cruwys T, Chang MX, Bentley SV, Haslam SA, Dingle GA, et al. GROUPS 4 HEALTH reduces loneliness and social anxiety in adults with psychological distress: findings from a randomized controlled trial. J Consult Clin Psychol. 2019 Sep;87(9):787801.

33 Bruce LD, Wu JS, Lustig SL, Russell DW, Nemecek DA. Loneliness in the United States: A 2018 National Panel Survey of Demographic, Structural, Cognitive, and Behavioral Characteristics. Am J Health Promot. 2019 Nov;33(8):1123-33.

34 Theeke LA, Mallow JA, Moore J, McBurney A, Rellick S, VanGilder R. Effectiveness of LISTEN on loneliness, neuroimmunological stress response, psychosocial functioning, quality of life, and physical health measures of chronic illness. Int J Nurs Sci. 2016 Sep; $3(3): 242-51$.
35 Lindsay EK, Young S, Brown KW, Smyth JM, Creswell JD. Mindfulness training reduces loneliness and increases social contact in a randomized controlled trial. Proc Natl Acad Sci USA. 2019 Feb;116(9):3488-93.

36 Zhang N, Fan FM, Huang SY, Rodriguez MA. Mindfulness training for loneliness among Chinese college students: A pilot randomized controlled trial. Int J Psychol. 2018 Oct;53(5): 373-8.

37 Choi NG, Pepin R, Marti CN, Stevens CJ, Bruce ML. Improving Social Connectedness for Homebound Older Adults: Randomized Controlled Trial of Tele-Delivered Behavioral Activation Versus Tele-Delivered Friendly Visits. Am J Geriatr Psychiatry. 2020 Jul; 28(7):698-708.

38 Guidi J, Brakemeier EL, Bockting CL, Cosci F, Cuijpers P, Jarrett RB, et al. Methodological Recommendations for Trials of Psychological Interventions. Psychother Psychosom. 2018; 87(5):276-84.

39 Andersson G. Internet-delivered psychological treatments. Annu Rev Clin Psychol. 2016;12(1):157-79.

40 Klein NS, Kok GD, Burger H, van Valen E, Riper H, Cuijpers P, et al. No Sustainable Effects of an Internet-Based Relapse Prevention Program over 24 Months in Recurrent Depression: Primary Outcomes of a Randomized Controlled Trial. Psychother Psychosom. 2018;87(1):55-7.

41 Donker T, Bennett K, Bennett A, Mackinnon $A$, van Straten $A$, Cuijpers $P$, et al. Internetdelivered interpersonal psychotherapy versus internet-delivered cognitive behavioral therapy for adults with depressive symptoms: randomized controlled noninferiority trial. J Med Internet Res. 2013 May;15(5):e82.

42 Dagöö J, Asplund RP, Bsenko HA, Hjerling S, Holmberg A, Westh S, et al. Cognitive behavior therapy versus interpersonal psychotherapy for social anxiety disorder delivered via smartphone and computer: a randomized controlled trial. J Anxiety Disord. 2014 May; 28(4):410-7.

43 Maes M, Qualter P, Vanhalst J, Van den Noortgate W, Goossens L. Gender differences in loneliness across the lifespan: A meta-analysis. Eur J Pers. 2019;33(6):642-54.

44 Hart T, Fann JR, Novack TA. The dilemma of the control condition in experience-based cognitive and behavioural treatment research. Neuropsychol Rehabil. 2008 Jan;18(1):1-21.

45 Colle R, Ait Tayeb AE, de Larminat D, Commery L, Boniface B, Chappell K, et al. Telepsychiatry in the post-COVID-19 era: Moving backwards or forwards? Psychother Psychosom. 2021;90(1):69-70. 